\title{
Correlation of BMI with breast cancer subtype and tumour size
}

\author{
K Govind Babu', Abhishek Anand'1, Kuntegowdanahalli C Lakshmaiah', Dasappa Lokanatha', Linu Abraham Jacob', \\ MC Suresh Babu', Kadabur N Lokesh'1 ${ }^{1}$ Haleshappa A Rudresha', Lakkavalli K Rajeev', Smitha C Saldanha ${ }^{1}$, GV Giri', \\ Chethan R' ${ }^{1}$, Deepak Koppaka', Dipti Panwar ${ }^{2}$ and Rekha V Kumar ${ }^{2}$
}

${ }^{1}$ Department of Medical Oncology, Kidwai Cancer Institute, Bengaluru 560029, India

${ }^{2}$ Department of Pathology, Kidwai Cancer Institute, Bengaluru 560029, India

Correspondence to: Abhishek Anand. Email: drabhishek2508@gmail.com

\section{Abstract}

Background: Breast cancer is a heterogeneous disease which is divided broadly into luminal, HER2 and basal type based on molecular profiling. Increased body mass index (BMI) has been associated with the risk of developing breast cancer but the association based on molecular subtype remains conflicting.

Methods: This was an observational study carried out over a period of 2 years. Nonmetastatic breast cancer patients were evaluated for the tumour subtype based on surrogate markers (ER, PR and HER2). The BMI of these patients was correlated with the tumour subtype and size.

Results: We studied 476 patients with breast cancer with the median age of 46 years (range, 25-86) and 58\% were premenopausal. The mean BMI of the cohort was 24.1, which was significantly higher in postmenopausal women (24.9 versus $23.6, p<0.05)$. Overall, only $10 \%$ of patients were obese. The mean BMI in the luminal, HER2 and TNBC subtypes was 24.7, 22.4 and 23.9, respectively ( $p<0.01$ ). Also, the mean tumour size in luminal, HER2 and TNBC subtype was $4.02,3.80$ and $4.27 \mathrm{~cm}$, respectively $(p=0.158)$.

Conclusion: The average BMI was higher in patients with luminal subtype followed by TNBC and lowest for HER2 at the time of diagnosis. The mean tumour size was numerically higher for TNBC and lowest for HER2 subtype although the difference was not statistically significant. Larger studies may provide clarity of association between the BMI and tumour subtype.

Keywords: body mass index, breast cancer

Published: 26/06/2018

Received: 03/02/2018

ecancer 2018, 12:845 https://doi.org/10.3332/ecancer.2018.845

Copyright: (C) the authors; licensee ecancermedicalscience. This is an Open Access article distributed under the terms of the Creative Commons Attribution License (http://creativecommons.org/licenses/by/3.0), which permits unrestricted use, distribution, and reproduction in any medium, provided the original work is properly cited. 


\section{Introduction}

Breast cancer is the most common cancer among females and is one of the commonest causes of cancer-related mortality worldwide [1]. In India, breast cancer is now the commonest cancer amongst women followed by carcinoma of the cervix with a rising incidence in young premenopausal women [2]. It is a well-known fact that breast cancer is a heterogeneous disease and has been divided into Luminal, HER2 enriched and basal-like subtype based on molecular profiling [3]. More recently, the genomic assays (oncotype DX, mammaprint, endopredict and Prosigna) are becoming a useful tool for defining the prognostic characteristics and in some cases for deciding the theraputic strategy. However, in routine clinical practice, breast cancer subtype is commonly approximated using the surrogate immunohistochemistry (IHC) markers, oestrogen receptor (ER), progesterone receptor (PR), human epidermal growth factor receptor 2(HER2) and Ki67 [4, 5]. An increased body mass index (BMI) has been found to be a risk factor for breast cancer in postmenopausal women. It is associated with more aggressive tumour biology and a poor prognosis $[6,7]$. There are few studies that have found high BMI to be associated with a higher proliferation index, histological grade, a larger tumour size and a higher number of axillary node metastasis at the time of diagnosis. Some studies have also related a higher incidence of ER-positive breast cancer with BMI [8, 9]. However, it remains uncertain whether the same association is present in the Indian population which has different reproductive patterns compared to a western population and possibly a different subtype distribution as well. Moreover, the prevalence of obesity is not similar as compared to the western population [10]. The present study aims to find any association between BMI, tumour subtype and the breast cancer tumour size.

\section{Methods}

This was an observational study of 476 breast cancer patients done at a tertiary cancer centre in India, from January 2016 to December 2017. Patients with a diagnosis of breast cancer and already having histopathology and IHC report of ER, PR, HER2 and Ki67 were included in the study. Patients underwent either modified radical mastectomy or breast conserving surgery depending on the stage. Those with in-situ carcinoma, nonductal pathology, bilateral breast cancer and metastatic disease were excluded from the study.

BMI was calculated using the Quetelet index using patient's height and weight $\left(\mathrm{BMI}=\right.$ weight in $\mathrm{kg} / \mathrm{height}$ in $\left.\mathrm{meter}{ }^{2}\right)$. The World Health Organization criteria were used to classify women based on BMI value into underweight and normal (BMI < 25); overweight $(\geq 25$ and $<30)$ and obese $(\geq 30)$ [11]. The tumour size measurement was based on the mammography and breast ultrasound. Imaging method was chosen for size criteria as a significant number of patients (32\%) presented with locally advanced disease and were planned for neoadjuvant chemotherapy which could have underestimated the tumour size on surgical specimen. Tumour subtyping was done based on the surrogate IHC markers, ER, PR, HER2 and Ki67. Allred scoring was used for hormone receptor (HR) status and the American Society of Clinical Oncology/College of American Pathologists (ASCO/CAP) guidelines 2013 was used for reporting HER2 [12, 13]. For HR, 3+ or more was taken positive while 3+ on IHC was taken positive for HER2. Those equivocal for HER2 $(2+$ on IHC) was confirmed by doing fluorescent in situ hybridization. The primary tumour subtyping was done based on surrogate IHC markers according to the St Gallen criteria 2013 [14]. It subdivided breast tumours into luminal (A and B), HER2 over-expressing and basal (triple negative, TNBC) subtypes.

The BMI was correlated with the breast cancer subtype as well as the tumour size. For comparing the means of different variables, $t$-test and ANOVA were used. A $p$ value of $<0.05$ was taken as significant. All analyses were done on SPSS, version 23.0 software.

\section{Results}

We studied 476 patients of breast cancer who fulfilled the inclusion criteria over a period of 2 years. The median age at diagnosis was 46 years (range, 25-82). At the time of diagnosis, 278 (58.4\%) patients were premenopausal whereas 198 (41.6\%) were postmenopausal. The average age of menarche and first live birth was 13.7 and 23.4 years, respectively. Four patients were nulliparous while the rest of the cohort had an average of 2.4 children (range, 1-7). Breastfeeding was done by 468 women (98.3\%). The mean BMI was $24.1 \mathrm{~kg} / \mathrm{m}^{2}$ with postmenopausal women having significantly higher BMI than premenopausal women (24.9 versus 23.6; $\left.p=0.02\right)$. Luminal type 
(53\%; including luminal A and B) was the most common subtype followed by TNBC (31\%) and HER2 enriched (16\%). The patient characteristics have been given in Table 1. Most of the patients (58\%) were in the BMI $<25$ group. Overweight (BMI 25-29.9) comprised 38\% of the patients, whereas only $10 \%$ were obese $(\mathrm{BMI} \geq 30$ ) in the present study. The mean BMI in the luminal, HER2 and TNBC subtypes were 24.7, 22.4 and 23.9, respectively $(p<0.01)$. The relation of BMI and the breast tumour subtype has been given in Table 2 . The tumour size according to the breast tumour subtype and BMI was also studied (Table 3). The mean tumour size in luminal, HER2 and TNBC subtype was $4.02,3.80$ and $4.27 \mathrm{~cm}$, respectively $(p=0.158)$. The mean tumour size for $\mathrm{BMI}<25$ was $3.99 \mathrm{~cm}$; for BMI 25-29.9, $4.13 \mathrm{~cm}$ and for $\mathrm{BMI} \geq 30$ the mean size was $4.30 \mathrm{~cm}(p=0.584)$.

Table 1. Patient characteristics $(n=476)$.

\begin{tabular}{|c|c|}
\hline Median age & 46 (range, 25-82) \\
\hline Premenopausal & $278(58 \%)$ \\
\hline Postmenopausal & $198(42 \%)$ \\
\hline Age of menarche (mean) & 13.7 (range, 11-17) \\
\hline Age at first live birth (mean) & 23.4 (range, 17-34) \\
\hline Number of children (mean) & 2.4 (range, $0-7$ ) \\
\hline Nulliparous & $4(1 \%)$ \\
\hline Breast feeding done & $468(98 \%)$ \\
\hline \multicolumn{2}{|l|}{ BMI $\left(\mathrm{kg} / \mathrm{m}^{2}\right)$} \\
\hline Mean & 24.1 (15.0-37.4, SD: 4.529) \\
\hline Premenopausal & 23.6 (15.0-37.4, SD: 4.507) \\
\hline postmenopausal & 24.9 (15.1-36.0, SD: 4.334) \\
\hline \multicolumn{2}{|l|}{ Tumour subtype } \\
\hline Luminal type & $252(53 \%)$ \\
\hline HER2 enriched & $77(16 \%)$ \\
\hline TNBC & $147(31 \%)$ \\
\hline \multicolumn{2}{|l|}{ T status (tumour) } \\
\hline T1 & $32(6.7 \%)$ \\
\hline T2 & $251(52.7 \%)$ \\
\hline T3 & $124(26.1 \%)$ \\
\hline T4 & $69(14.5 \%)$ \\
\hline \multicolumn{2}{|l|}{ N status (node) } \\
\hline No & $136(28.6 \%)$ \\
\hline $\mathrm{N}+$ & $340(71.4 \%)$ \\
\hline \multicolumn{2}{|l|}{ Stage } \\
\hline I & 17 \\
\hline II & 212 \\
\hline III & 247 \\
\hline
\end{tabular}


Table 2. Number of patients according to BMI in each subtype of breast cancer.

\begin{tabular}{|l|c|c|c|c|}
\hline \multicolumn{5}{|c|}{ Number of patients in each subgroup } \\
\hline \multicolumn{1}{|c|}{ BMI } & Luminal $(\boldsymbol{n}=\mathbf{2 5 2})$ & HER2 $(\boldsymbol{n}=\mathbf{7 7})$ & TNBC $(\boldsymbol{n}=\mathbf{1 4 7})$ & Total $(\boldsymbol{n}=\mathbf{4 7 6})$ \\
\hline$<25$ & $125(49 \%)$ & $64(83 \%)$ & $88(60 \%)$ & $277(58 \%)$ \\
\hline $25-<30$ & $98(39 \%)$ & $11(14 \%)$ & $4329 \%)$ & $152(38 \%)$ \\
\hline$\geq 30$ & $29(12 \%)$ & $2(3 \%)$ & $16(11 \%)$ & $47(10 \%)$ \\
\hline Mean BMI & $24.7(15.0-37.4$, SD:4.628) & $22.4(15.1-33.0$, SD:3.711) & $23.9(17.5-37.5$, SD:4.361) & $24.1(15.0-37.4$, SD: 4.529$)$ \\
\hline
\end{tabular}

Table 3. Mean tumour size in cms according to BMI and subtype.

\begin{tabular}{|l|c|c|c|c|}
\hline $\begin{array}{c}\text { BMI }(\mathbf{k g} / \\
\left.\mathbf{m}^{2}\right)\end{array}$ & Luminal & HER2 & TNBC & Total \\
\hline$<25$ & $3.89(1.5-11.0 ;$ SD: 1.886$)$ & $3.77(1.6-10.0 ;$ SD: 1.912$)$ & $4.29(2.0-9.0 ;$ SD: 1.484$)$ & $3.99(1.5-11.0 ;$ SD: 1.781$)$ \\
\hline $25-<30$ & $4.1(1.5-10.0 ;$ SD: 2.103$)$ & $3.94(1.9-6.2 ;$ SD: 1.564$)$ & $4.15(1.5-8.0 ;$ SD: 1.745$)$ & $4.13(1.5-10.0 ;$ SD:1.963) \\
\hline$\geq 30$ & $4.2(1.5-7.4 ;$ SD: 1.554$)$ & $4.0(3.5-4.5 ;$ SD: 0.707$)$ & $4.5(2.5-7.5 ;$ SD: 1.811$)$ & $4.3(1.5-7.5$, SD: 1.605$)$ \\
\hline Mean $(\mathrm{cm})$ & $4.02(1.5-11.0 ;$ SD:1.936) & $3.80(1.6-10.0 ;$ SD: 1.834$)$ & $4.27(1.5-9.0 ;$ SD: 1.592$)$ & $4.06(1.5-11.0 ;$ SD: 1.823$)$ \\
\hline
\end{tabular}

\section{Discussion}

We observed a higher BMI for luminal subtype at diagnosis followed by TNBC and HER2 subtype. Also, a higher BMI was associated with a larger tumour at presentation. TNBC subtype was associated with the maximum tumour size followed by luminal and HER2 in the present study. The exact relation of BMI and breast cancer remains conflicting even after numerous studies. Postmenopausal obesity has been a known risk factor for the occurrence of breast cancer [15]. However, there are studies in which higher BMI has been related to breast cancer risk in premenopausal women but not in postmenopausal women [16]. In a meta-analysis, the clinical importance of obesity on breast cancer risk was not seen [17]. There are studies showing a positive correlation of BMI with tumour size, stage and grade of the tumour [18-22]. BMI and its relation to the breast cancer subtype have also been studied, but with conflicting data. In a large retrospective analysis of 3,767 breast cancer patients, authors concluded that TNBC subtype had a significant association with premenopausal obesity [22]. Similar conclusions were made by other studies as well that analysed the BMI and its association with breast cancer subtype [23-25]. However, there are studies which have shown a more likely association of obesity with hormone positive breast cancer [26-29]. In the present study, HER2 (83\%) and TNBC (60\%) subtype have a higher proportion of women with BMI < 25 compared to the luminal subtype (49\%). On the contrary, the proportion of patients with $\mathrm{BMI} \geq 25$ was higher in the luminal subtype (51\%) compared to TNBC (40\%) and HER2 (17\%).

The clinical identification of the tumour is delayed in obese patients, which results in a larger tumour size at the time of diagnosis [30]. This has been shown in studies done by Daling et al [8], Loi et al [9], and Abrahamson et al [31]. In another large study by Biglia et al [19] on 2,148 women of breast cancer, there was a significant association of a higher BMI with larger tumour size at presentation. In this study, $45 \%$ of postmenopausal women with BMI > 25 had a tumour measuring more than $2 \mathrm{~cm}$, compared to $33.4 \%$ in normal and $21 \%$ in underweight women. The mean tumour size in the present study for women with BMI < 25 was $3.99 \mathrm{~cm}$; for BMI $25-29.9$ was $4.13 \mathrm{~cm}$ and for BMI $\geq$ 30 was $4.30 \mathrm{~cm}$. On tumour subtyping, the mean tumour diameter was maximum for TNBC (4.27 cm) and minimum for HER2 (3.80 cm).

In a study done by Castaneda et al [32], the TNBC subtype was more likely to present with a larger tumour when compared to luminal or HER2 subtype. They also found no significant difference in the T stage for the HER2 subtype. The present study shows that the aggressive subtype of breast cancer (HER2 and TNBC) was more likely to develop in nonoverweight women compared to the luminal subtype, which was more common in overweight and obese women. Also, the mean tumour diameter was maximum for TNBC and minimum for the HER2 subtype. 
It is important to know the BMI, as many studies have shown a poor outcome associated with obese breast cancer patients [33-35]. Although this observation is fairly similar for luminal subtype, the outcomes for other subtypes remain conflicting. In a recent study using PAM-50 to define breast cancer subtype, the association of BMI with outcome was not dependent on the defined molecular subtype [36].

The biologic mechanism behind poor outcomes of breast cancer patients associated with obesity is multifactorial. Metabolic factors of obesity, e.g. insulin, glucose and adipokines like leptin, and inflammatory factors have been related to the poor outcomes. Among various factors, insulin plays an important role by binding to the insulin receptors expressed on breast cancer cells and activate signaling through PI3K/Akt and Ras/MAPK pathways, which finally leads increased tumour proliferation [37, 38].

Although obesity is associated with worse outcome, data pertaining to the effect of weight loss on outcomes after diagnosis is inconsistent. However, counselling for weight loss, lifestyle modification, physical activity and nutrition should be given to all breast cancer patients to maintain a healthy weight. The rationale for maintaining a healthy weight is for general health benefit as a high-level evidence of improving outcomes with weight control after diagnosis is still lacking.

\section{Conclusion}

In conclusion, the present study shows that patients with luminal subtype breast cancer have the highest BMI at diagnosis followed by TNBC and HER2 subtypes. Higher BMI was associated with larger tumour at presentation and TNBC having the maximum tumour size followed by luminal and HER2. Large prospective studies are warranted for a better understanding of the association between the BMI and tumour characteristics, molecular subtype, as well as treatment outcomes.

\section{References}

1. World Health Organisation (2015) GLOBOCAN 2012 Geneva: World Health Organisation [http://globocan.iarc.fr/Pages/fact sheets cancer.aspx] Date accessed: 20/01/18

2. Mallath MK, Taylor DG, and Badwe RA, et al (2014) The growing burden of cancer in India: epidemiology and social context Lancet Oncol 15(6) e205-e212 https://doi.org/10.1016/S1470-2045(14)70115-9 PMID: 24731885

3. Sørlie T, Perou CM, and Tibshirani R, et al (2001) Gene expression patterns of breast carcinomas distinguish tumor subclasses with clinical implications Proc Natl Acad Sci USA 98 10869-10874 https://doi.org/10.1073/pnas.191367098 PMID: 11553815 PMCID: $\underline{58566}$

4. Perou CM, Sørlie T, and Eisen MB, et al (2000) Molecular portraits of human breast tumours Nature 406 747-752 https://doi. org/10.1038/35021093 PMID: 10963602

5. Carey LA, Perou CM, and Livasy CA, et al (2006) Race, breast cancer subtypes, and survival in the Carolina Breast Cancer Study JAMA 295 2492-2502 https://doi.org/10.1001/jama.295.21.2492 PMID: 16757721

6. Renehan AG, Tyson M, and Egger M, et al (2008) Body-mass index and incidence of cancer: a systematic review and meta-analysis of prospective observational studies Lancet 371 569-578 https://doi.org/10.1016/S0140-6736(08)60269-X PMID: 18280327

7. Petrelli JM, Calle EE, and Rodriguez C, et al (2002) Body mass index, height, and postmenopausal breast cancer mortality in a prospective cohort of US women Cancer Causes Control 13 325-332 https://doi.org/10.1023/A:1015288615472 PMID: 12074502

8. Daling JR, Malone KE, and Doody DR, et al (2001) Relation of body mass index to tumor markers and survival among young women with invasive ductal breast carcinoma Cancer 92 720-729 PMID: 11550140 
9. Loi S, Milne RL, and Friedlander ML, et al (2005) Obesity and outcomes in premenopausal and postmenopausal breast cancer Cancer Epidemiol Biomarkers Prev 14 1686-1691 https://doi.org/10.1158/1055-9965.EPI-05-0042 PMID: 16030102

10. World Health Organization (2018) Obesity and overweight Geneva: World Health Organization [http://www.who.int/mediacentre/ factsheets/fs311/en/] Date accessed: 20/01/18

11. World Health Organization (WHO) (2008) WHO, global database on body mass index, BMI classification 2 Geneva: WHO [http:/l apps.who.int/bmi/index.jsp?introPage=intro 3.html]

12. Allred DC, Bustamante MA, and Daniel CO, et al (1990) Immunocytochemical analysis of estrogen receptors in human breast carcinomas. Evaluation of 130 cases and review of the literature regarding concordance with biochemical assay and clinical relevance Arch Surg 125 107-113 https://doi.org/10.1001/archsurg.1990.01410130113018 PMID: 1688490

13. (2013) Updated guideline on HER2 testing released by CAPIASCO PharmacoEconomics \& Outcomes News 689(1) 3 https://doi. org/10.1007/s40274-013-0779-6

14. Goldhirsch A, Winer EP, and Coates AS, et al (2013) Personalizing the treatment of women with early breast cancer: highlights of the St Gallen International Expert Consensus on the Primary Therapy of Early Breast Cancer 2013 Ann Oncol 24(9) 22062223 https://doi.org/10.1093/annonc/mdt303 PMID: 23917950 PMCID: $\underline{3755334}$

15. Peng X, Li JG, and Feng J, et al (2013) Prognostic significance of body mass index in breast cancer patients with hormone receptor-positive tumours after curative surgery Clin Invest Med 36(6) 297e305

16. Cecchini RS, Costantino JP, and Cauley JA, et al (2012) Body mass index and the risk for developing invasive breast cancer among high-risk women in NSABP P-1 and STAR breast cancer prevention trials Cancer Prev Res 5(4) 583e92 https://doi. org/10.1158/1940-6207.CAPR-11-0482

17. Cheraghi Z, Poorolajal $\mathrm{J}$, and Hashem $\mathrm{T}$, et al (2012) Effect of body mass index on breast cancer during premenopausal and postmenopausal periods: a meta-analysis PLoS One 7(12) e51446 https://doi.org/10.1371/journal.pone.0051446 PMID: 23236502 PMCID: $\underline{3517558}$

18. Eichholzer M, Huang DJ, and Modlasiak A, et al (2013) Impact of body mass index on prognostically relevant breast cancer tumor characteristics Breast Care 8(3) 192e8 https://doi.org/10.1159/000350002

19. Biglia N, Peano E, and Sgandurra P, et al (2013) Body mass index (BMI) and breast cancer: impact on tumor histopathologic features, cancer subtypes and recurrence rate in pre and postmenopausal women Gynecol Endocrinol 29(3) 263e7 https://doi. org/10.3109/09513590.2012.736559

20. Chen X, Lu W, and Zheng W, et al (2010) Obesity and weight change in relation to breast cancer survival Breast Cancer Res Treat 122(3) 823e33 https://doi.org/10.1007/s10549-009-0708-3

21. Rosenberg L, Czene K, and Hall P (2009) Obesity and poor breast cancer prognosis: an illusion because of hormone replacement therapy? Br J Cancer 100(9) 1486e91 https://doi.org/10.1038/sj.bjc.6605025

22. Sahin S, Erdem GU, and Karatas F, et al (2016) The association between body mass index and immunohistochemical subtypes in breast cancer Breast 32 227-236 https://doi.org/10.1016/j.breast.2016.09.019 PMID: 27756509

23. Turkoz FP, Solak M, and Petekkaya I, et al (2013) The prognostic impact of obesity on molecular subtypes of breast cancer in premenopausal women $J$ BUON 18(2) 335e41

24. Petekkaya I, Sahin U, and Gezgen G, et al (2013) Association of breast cancer subtypes and body mass index Tumori 99(2) 129e33

25. Maehle BO, Tretli S, and Skjaerven R, et al (2001) Premorbid body weight and its relations to primary tumour diameter in breast cancer patients; its dependence on estrogen and progesteron receptor status Breast Cancer Res Treat 68(2) 159e69 https://doi. org/10.1023/A:1011977118921 
26. Yang XR, Chang-Claude J, and Goode EL, et al (2011) Associations of breast cancer risk factors with tumor subtypes: a pooled analysis from the Breast Cancer Association Consortium studies J Natl Cancer Inst 103(3) 250e63 https://doi.org/10.1093/jnci/dja526

27. Enger SM, Ross RK, and Paganini-Hill A, et al (2000) Body size, physical activity, and breast cancer hormone receptor status: results from two case-control studies Cancer Epidemiol Biomark Prev 9(7) 681e7

28. Colditz GA, Rosner BA, and Chen WY, et al (2004) Risk factors for breast cancer according to estrogen and progesterone receptor status J Natl Cancer Inst 96(3) 218e28 https://doi.org/10.1093/jnci/djh025

29. Suzuki R, Orsini N, and Saji S, et al (2009) Body weight and incidence of breast cancer defined by estrogen and progesterone receptor statusea meta-analysis Int J Cancer J Int du Cancer 124(3) 698e712 https://doi.org/10.1002/ijc.23943

30. Cui Y, Whiteman MK, and Flaws JA, et al (2002) Body mass and stage of breast cancer at diagnosis Int J Cancer 98 279-283 https://doi.org/10.1002/ijc.10209 PMID: 11857420

31. Abrahamson PE, Gammon MD, and Lund MJ, et al (2006) General and abdominal obesity and survival among young women with breast cancer Cancer Epidemiol Biomarkers Prev 15 1871-1877 https://doi.org/10.1158/1055-9965.EPI-06-0356 PMID: 17035393

32. Castaneda CA, Andrés E, and Barcena C, et al (2012) Behaviour of breast cancer molecular subtype through tumour progression Clin Trans/ Oncol 14481 https://doi.org/10.1007/s12094-012-0827-x PMID: 22634538

33. Protani M, Coory M, and Martin JH (2010) Effect of obesity on survival of women with breast cancer: systematic review and meta-analysis Breast Cancer Res Treat 123 627-635 https://doi.org/10.1007/s10549-010-0990-0 PMID: 20571870

34. Niraula S, Ocana A, and Ennis M, et al (2012) Body size and breast cancer prognosis in relation to hormone receptor and menopausal status: a meta-analysis Breast Cancer Res Treat 134 769-781 https://doi.org/10.1007/s10549-012-2073-x PMID: 22562122

35. Chan DS, Vieira AR, and Aune D, et al (2014) Body mass index and survival in women with breast cancer: Systematic literature review and meta-analysis of $\mathbf{8 2}$ follow-up studies Ann Oncol 25 1901-1914 https://doi.org/10.1093/annonc/mdu042 PMID: 24769692 PMCID: 4176449

36. Ligibel JA, Cirrincione CT, and Liu M, et al (2015) Body mass index, PAM50 subtype, and outcomes in nodepositive breast cancer: CALGB 9741 (Alliance) J Natl Cancer Inst 25107

37. Goodwin PJ and Stambolic V (2015) Impact of the obesity epidemic on cancer Annu Rev Med 66 281-296 https://doi.org/10.1146/ annurev-med-051613-012328

38. Goodwin PJ, Ennis M, and Pritchard KI, et al (2012) Insulin- and obesity-related variables in early-stage breast cancer: correlations and time course of prognostic associations J Clin Oncol 30 164-171 https://doi.org/10.1200/JC0.2011.36.2723 